\title{
One Case Report and Literature Review of Alexander's Disease Caused by a Mutation in the GFAP Gene
}

\author{
Gaohong $\mathrm{Wu}^{1}{ }^{1}$ Xueping Zhu ${ }^{* 1}$ and Ping Jiang ${ }^{2}$ \\ ${ }^{1}$ Department of Neonatology, Children's Hospital of Soochow University, China \\ ${ }^{2}$ Bayingolin Mongolian autonomous prefecture people's hospital, China
}

*Corresponding author: Xueping Zhu, Department of Neonatology, Children's Hospital of Soochow University, China.

To Cite This Article: Xueping Zhu, Gao Hong Wu, Ping Jiang.One Case Report and Literature Review of Alexander's Disease Caused by a Mutation in the GFAP Gene. Am J Biomed Sci \& Res. 2019 - 6(2). AJBSR.MS.ID.001019. DOI: 10.34297/AJBSR.2019.06.001019.

Received: 眥 November 08, 2019; Published: 些 November 18, 2019

\begin{abstract}
Background: Based on a case of tic as the primary symptom of Alexander disease I type, enhancing understanding clinical manifestations and characteristics of the genotypes and improving the level of diagnosis and treatment of Alexander disease I type.

Methods: Combined one with gene diagnosed type I Alexander disease child in October 2018 in admission to neonatal ward of Children's Hospital of Soochow University, the clinical diagnosis and treatment process of patient and related literature reports were analyzed retrospectively, follow-up was conducted in February 2019, the etiology, clinical manifestations, genotypic characteristics, diagnosis and treatment were discussed.

Results: One 20-day-old boy, the main symptom was convulsion, improve related laboratory inspection, finally, GFAP gene detection was passed, (ngqx1801877701-1) chr17: 42989062 showed heterozygous mutation of c.884A >G (p.d295g), It has not been reported at home or abroad, and the child can be diagnosed with Alexander disease I type, moreover, the father of the patient had heterozygous mutation (ngqx1801877802-1) chr17: 42989062 with c.844A>G (p.d295g), after the treatment of symptomatic support, the patient's condition improved.
\end{abstract}

Conclusion: Alexander disease I type early disease, early diagnosis and under the support in symptomatic treatment, attaches great importance to the late follow-up and rehabilitation training is the key to improve the prognosis.

Keywords: Alexander’s disease type I; Convulsions; GFAP gene; Follow-up visit

\section{Background}

Alexander disease (AxD), the first known hereditary astrocyte disease, is a rare progressive leukoencephalopathy, which is an autosomal dominant genetic disease caused by mutation of GFAP gene encoding glial fibrillary acidic protein. According to the age of onset and clinical manifestations, it can be divided into two types: type I (onset age $<4$ years) mainly manifest as large head circumference, epileptic seizures, developmental retardation and paroxysmal aggravation, type II has late onset, manifested as autonomic dysfunction, eye movement abnormalities, palatal myoclonus and medulla oblongata symptoms [1]. Before the discovery of the pathogenic gene of $\mathrm{AxD}$, the diagnosis was mainly based on the pathological biopsy of brain tissue [2]. The clinical manifestations, genotype characteristics and follow-up of children with type I AxD diagnosed by GFAP gene analysis and clinical characteristics in the Children's Hospital of Soochow University in 2018 were retrospectively analyzed.

\section{Case Presentation}

A male patient, 20 days old, was admitted to hospital on September 27, 2018 because of "Extremities convulsions were found for 18 days". The patient belonged to cesarean section, with $36^{+6}$ weeks of gestational age, no history of hypoxia and asphyxia after birth, birth weight of $2500 \mathrm{~g}$, gestational diabetes mellitus during pregnancy, extremities convulsions after birth, and more and more frequent convulsions in the past 18 days. The parents of the child denied the consanguineous marriage and the family history of genetic diseases. Physical examination: body weight for $3.16 \mathrm{~kg}$, body length for $55 \mathrm{~cm}$, head circumference for $52.5 \mathrm{~cm}$, normal reaction, no special face, normal hair color and distribution, plain and soft anterior fontanel with about $2.0 \times 2.0 \mathrm{~cm}$ size, soft neck, no abnormality in heart, lung and abdomen examination, normal limb movement, normal muscular tension, negative pathological signs. Laboratory examination: blood routine, urine routine, stool 
routine, thyroid function, coagulation function, electrolyte, renal function, blood ammonia, blood lactic acid, TORCH, blood glucose were in the normal range; hereditary metabolism of hematuria: decreased arginine; electroencephalogram: mildly abnormal electroencephalogram; cranial MRI: cranial MRI showed neonatal brain changes.

\section{Molecular Genetics}

After the parents signed the informed consent, $5 \mathrm{ml}$ of whole peripheral blood with EDTA was taken from the patient and their parents, and genomic DNA was extracted. Gene analysis related to metabolic diseases and leukoencephalopathy was carried out by using the genetic metabolic diseases and leukoencephalopathy detection program from Beijing Running Gene Co., Ltd. There was a heterozygous mutation of c.884A $>$ G (p.D295G) in child with GFAP gene ((NGQX1801877701-1) chr17: 42989062), a heterozygous mutation of c.844A $>$ G (p.D295G) was found in father-carried NGQX1801877802-1 (chr17:42989062) (Figure 1), and no mutation was found in mother. By searching the human mutation gene database (HMGD) and PubMed database, it was found that the mutation has not been reported at home and abroad, which is a new mutation. The same loci variation was not found by further inquiries for HGM Dpro database. The mechanism of AxD caused by the mutation of GFAP gene has been identified, the mutation can be judged as pathogenic mutation according to the guidelines of the American College of Medical Genetics and Genomics (ACMG). Therefore, the mutation can be judged as a pathogenic mutation. Therefore, the molecular genetic diagnosis of the child was clear, eventually diagnosed as type I AxD. The father of the child was a carrier with heterozygous mutation, of which childhood had similar performance with the child. The genetic pattern of this gene is dominant inheritance, which conforms to the family co-segregation.

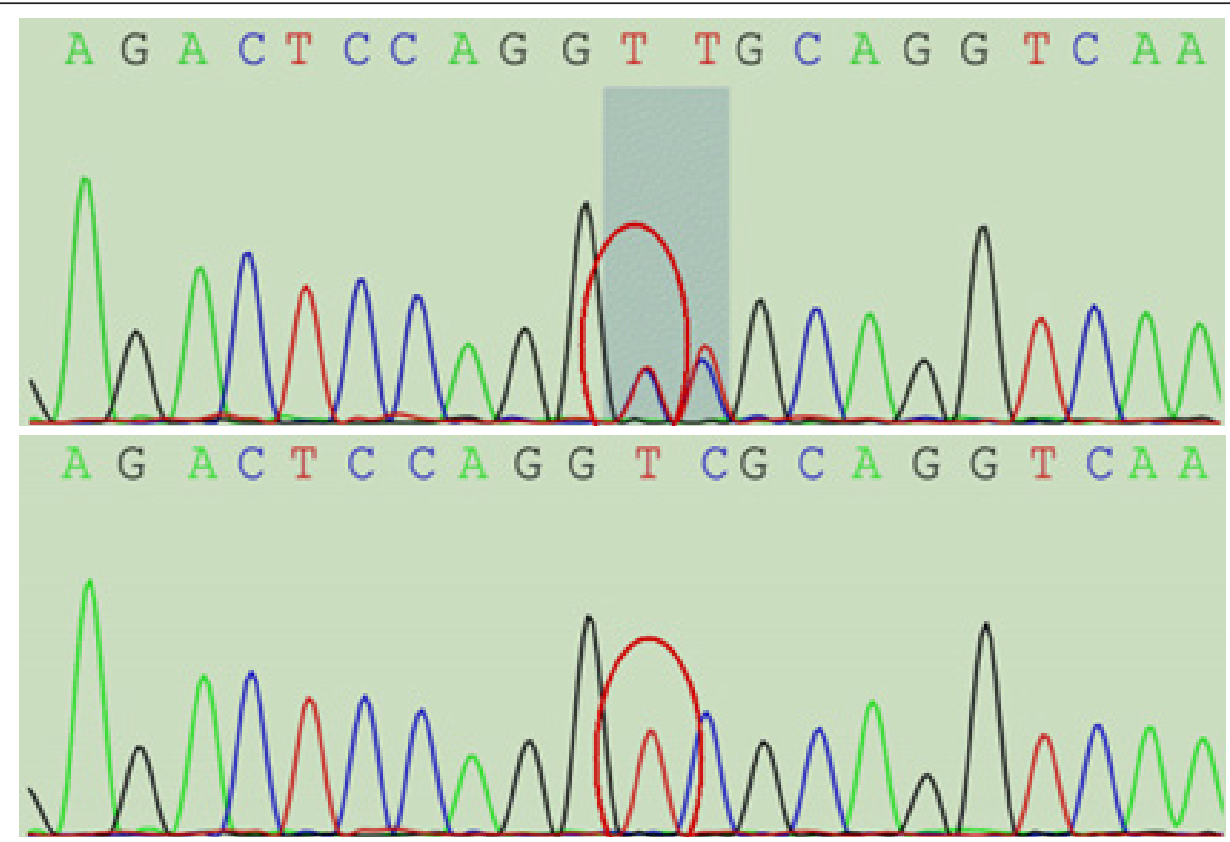

Figure 1: Sanger Sequencing of GFAP Gene in Child with Type I AxD and his Parents.

Note: The red circle shows the mutation position of c.884A > G; A: c.884A > G mutation is detected in GFAP gene of child; B: c.884A > G mutation is detected in GFAP gene of father; C: c.884A > G mutation is not detected in GFAP gene of mother.

\section{Treatment and Follow-up}

The child was a newborn with convulsion as the main manifestation, of which head circumference was large and the level of serum arginine was decreased. Combined with cranial MRI and heterozygous mutation of GFAP gene, the child was diagnosed as type I AxD. Vitamin B6 nourishing nerve and other supportive treatment, as well as targeted behavior and development training for child by the parents, the rehabilitation treatment was carried out and the curative effect was acceptable. In February 2019, telephone follow-up showed that the child was 6 months old, weighed 8,000 g, had no convulsions, could raise his head and turn over, and could not sit alone.

\section{Discussion}

AxD, also known as Fibrinnoid leukodystrophy, is a rare nonfamilial lethal leukoencephalopathy, which may be autosomal dominant genetic disease, mostly sporadic cases [3]. This disease is associated with multiple GFAP gene mutations, which is a leukodystrophy caused by astrocyte dysfunction caused by GFAP gene mutations. The mutant GFAP may impair the function of astrocyte by blocking normal intermediate filament polymerization, resulting in fatal lesions of white matter, of which the main pathological features were Rosenthal fiber aggregation and demyelination changes. The main clinical manifestations of AxD were epileptic seizure, squared skull, retardation of development, 
medulla oblongata symptoms, ataxia and neurodegenerative lesions. According to the age of onset and clinical manifestations, AxD can be divided into infant type ( $<2$ years old), juvenile type (2-12 years old) and adult type ( $>12$ years old) [4].

The first visit age of the child is 20 days after birth, which conforms to the characteristics of early onset (usually $<4$ years old) of patient with type I AxD reported internationally [1]. Because most of children with type I AxD (79\%) are underdeveloped from childhood with concealed onset, the description of the type I AxD onset age is not accurate, and the course of disease cannot be simply calculated from the "onset" described by their parents. Epilepsy seizures enlarged head circumference and developmental retardation are common clinical manifestations of type I AxD, epilepsy seizures are common clinical manifestations for both infant type springing from traditional typing and type I AxD [5]. The motor function is often affected in children with this disease, which is also consistent with the manifestation of white matter with serious involvement on skull MRI. Epilepsy seizure is a common clinical manifestation in infant type springing from traditional typing, with epilepsy incidence of $92 \%$ [6]. In this case, convulsions were the main complaint and the onset age was early, which might be related to the over-excitation of neurons caused by decreased glutamate reuptake ability of astrocytes [7]. Head circumference enlargement was more common in type I AxD, and the case was significantly enlarged, which was consistent with it [1]. Generally, the abnormal signal of white matter in AxD patients is diffuse and symmetrical on cranial MRI, which shows long T1 and long T2 signals. First, it appears in bilateral frontal lobes, while subcortical arcuate fibers are not involved. The lesion extends progressively back to parietal lobe, internal capsule area, occipital lobe, cerebellum and white matter of brainstem [8]. The electroencephalogram indicated abnormal electroencephalogram (EEG), and skull MRI showed underdeveloped brain changes, which were like MRI findings in the children with AxD.

\section{The key points of AxD diagnosis mainly include}

a) AxD can occur at all ages, especially in infants;

b) Clinical manifestations are aponea, epilepsy, myoclonus, bulbar paralysis, pyramidal sign and signs and symptoms of cerebellar damage, and mental and motor retardation in infants and adolescents;

c) Imaging manifestations were extensive white matter abnormalities predominantly in the frontal lobe or symmetrical lesions involving brainstem, cerebellum and spinal cord white matter, accompanied by enlargement or atrophy of medulla oblongata and spinal cord;

d) Metabolic screening (blood lactic acid, pyruvate, long chain fatty acid, thyrotropin, $\mathrm{N}$-acetyl aspartic acid, arylsufatase A, $\beta$-galactosidase, etc.) was normal. e) No other family history of leukodystrophy;

f) Mutations in GFAP gene were found by molecular biology examination;

g) Normal karyotype;

h) Rosenthal fibers were confirmed by brain biopsy [7].

The patient was a newborn with convulsion as the main manifestation, large head circumference and brain retardation diagnosed by MRI. Combined with GFAP gene detection, there was a heterozygous mutation of c.884A $>$ G (p.D295G) in (NGQX1801877701-1) chr17:42989062, so it was diagnosed as type I AxD. GFAP gene is the only pathogenic gene known at present, of which more than 100 mutations have been reported in the world. Except for a few small deletion and insertion mutations [7], most mutations are heterozygous missense mutations [6]. There was a heterozygous mutation of c.884A $>$ G (p.D295G) in child with NGQX1801877701-1 (chr17: 42989062), and a heterozygous mutation of c.844A $>$ G (p.D295G) was found in father-carried NGQX1801877802-1 (chr17:42989062), which was consistent with it. At present, four mutation hotspots have been reported in the world, including p. Arg239 (20.3\%), p. Arg79 (16.6\%), p. Arg88 (7.9\%) and p. Arg416 (5.6\%).The mutation point of this case is p.D295G, which has not been reported at home and abroad, and may be a rare mutation point. This disease should be distinguished from canavan disease (CD), both of which can be characterized by enlarged head circumference in infancy, retardation of autonomic nervous function, visual impairment, convulsions, progressive regression of intelligence and motor in preschool or school age. In MRI, if white matter lesions develop symmetrically backward from frontal lobe, AxD should be considered more. If white matter dispersively and symmetrically develops towards the center, it may be CD. N-acetylaspartic acid in laboratory blood and urine is not high when it is AxD, and high when it is CD. Combined with the history of children, MRI features and gene detection, the diagnosis can be clearly confirmed. At present, there is no effective treatment for AxD. The child received vitamin B6 nourishing nerve and other supportive treatment and rehabilitation training, with no convulsions and acceptable motor development.

To sum up, AxD belongs to an autosomal dominant genetic disease. The clinical characteristics of type I AxD are recurrent seizures, developmental retardation, enlarged head circumference and paroxysmal aggravation. A few children also have mental disorders, brainstem symptoms, idiopathic scoliosis or kyphosis. The diagnosis is mainly based on clinical manifestations, skull MRI and gene detection. There is no specific treatment for this disease, and more attention should be paid to late rehabilitation training under the premise of symptomatic and supportive treatment. At present, animal experiments have proved that phosphoinositide 3-kinase and quercetin can reduce the expression of GFAP, but no clinical pharmacological test has been carried out, and it is expected 
to be used in patients with mutation of GFAP [9]. There are also reports that Anti-aging gene activation may be relevant to prevent and treat neuron associated mental retardation and assist with motor development in AxD. Compounds such as resveratrol (antiaging gene activator) may modulate GFAP from cells in the brain to assist with the treatment of mental retardation, therefore, do the alterations in astrocyte (GFAP expression) induce neuronal deficits in patients with AxD still needs further research [10,11]. Most of the children with type I AxD have motor dysfunction after 7 years old, and this case is young and has no obvious motor dysfunction, so subsequent follow-up summary was needed. The characteristics of genotype I AxD are mostly new heterozygous missense mutations. In this case, the new mutation c.884A>G (p.D295G) of type I AxD and its GFAP gene enriched the mutation spectrum of AxD. The molecular genetic diagnosis of the proband is of great significance to the family of the sick child and is an important basis for genetic counseling and risk assessment of the disease. More cases should be collected, and clinical follow-up studies should be continued.

\section{Conclusion}

Alexander disease I type early disease, early diagnosis and under the support in symptomatic treatment, attaches great importance to the late follow-up and rehabilitation training is the key to improve the prognosis.

\section{Declarations}

Ethics approval and consent to participate. This study was reviewed and approved by the Ethics Committee of the Children's Hospital of Suzhou University (Suzhou, Jiangsu, China).

\section{Funding}

This study was financially supported by the following grants: The National Natural Science Foundation of China (NSFC, No. 81771626), The Jiangsu Provincial Maternal and Child Health Key Talents Project (No. FRC201731), and The Project of Science and Technology Development Plan of Suzhou (No. SS201428), Diagnosis and Treatment Technology Project of Clinical Key Diseases in Suzhou province (No. LCZX201612), and Minsheng TechnologyKey Technology Application Research Project (No. SS201644).

\section{Author Contributions}

GW, PJ wrote the manuscript, XZ participated in data analysis, interpretation of data and revising of the manuscript. All authors read and approved the final manuscript.

\section{References}

1. Prust M, Wang J, Morizono H, Messing A, Brenner M, et al. (2011) GFAP mutations, age at onset, and clinical subtypes in Alexander disease. Neurology 77(13): 1287-1294.

2. Messing A, Brenner M, Feany MB, Nedergaard M, Goldman JE (2012) Alesander disease. J Neurosci, 32(15): 5017-5023.

3. Johnson AB (2002) Alexander disease: A review and the gene International Journal of Developmental Neuroscience 20(3): 391-394.

4. Messing A, Goldman JE, Johnson AB, Michael Brenner (2001) Alexander disease: new insights from genetics. J Neuropathol Exp Neurol 60(6): 563-573.

5. Zang L, Wang J, Jiang Y, Qiang Gu, Zhijie Gao, et al. (2013) Follow-up study of 22 Chinese children with Alexander disease and analysis of parental origin of de novo GFAP mutations. Journal of Human Genetics 58(4): 183-188.

6. Rong LM, Johnson AB, Salomons G, James E Goldman, Sakkubai Naidu, et al. (2005) Glial fibrillary acidic protein mutations in infantile, juvenile, and adult forms of Alexander disease. Annals of Neurology 57(3): 310326.

7. Tian R, Wu X, Hagemann TL, Sosunov AA, Messing A, et al. (2010) Alexander disease mutant glial fibrillary acidic protein compromises glutamate transport in astrocytes. J Neuropathol Exp Neurol 69(4): 335345 .

8. Brenner M, Johnson AB, Boespflugtanguy $\mathrm{O}$, Rodriguez D, Goldman JE, et al. (2001) Mutations in GFAP, encoding glial fibrillary acidic protein, are associated with Alexander disease. Nature Genetics 27(1): 117-120.

9. Roymans D, Vissenberg K, Jonghe CD, Grobben B, Claes P, et al. (2001) Phosphatidylinositol 3-kinase activity is required for the expression of glial fibrillary acidic protein upon cAMP-dependent induction of differentiation in rat C6 glioma. Journal of Neurochemistry 76(2): 610618.

10. Ian James Martins (2016) Anti-Aging Genes Improve Appetite Regulation and Reverse Cell Senescence and Apoptosis in Global Populations. Advances in Aging Research 5(1): 9-26.

11. Ian J Martins (2017) Nutrition Therapy Regulates Caffeine Metabolism with Relevance to NAFLD and Induction of Type 3 Diabetes. J Diabetes Metab Disord 4: 019. 